\title{
Efeito da cisaprida e da fisioterapia respiratória sobre o refluxo gastroesofágico de lactentes chiadores segundo avaliação cintilográfica
}

\author{
Effects of cisapride and chest physical therapy on the gastroesophageal reflux \\ of wheezing babies based on scintigraphy
}

\author{
Maria Angela G.O. Ribeiro ${ }^{1}$, Marcelo Livorsi da Cunha $^{2}$, Elba Cristina de Camargo Etchebehere ${ }^{2}$, \\ Edwaldo E. Camargo ${ }^{3}$, José D. Ribeiro ${ }^{4}$, Antônio Condino-Neto ${ }^{4}$
}

\section{Resumo}

Objetivo: analisar o efeito da cisaprida e da fisioterapia respiratória em lactentes chiadores (LC), com doença do refluxo gastroesofágico (DRGE).

Métodos: avaliamos, prospectivamente, em 13 LC com DRGE e 12 sem DRGE, a densidade nuclear de tecnécio $\left({ }^{99} \mathrm{Tc}\right)$ em 3 topografias esofágicas. Os 2 grupos foram submetidos a investigação clínica, exames laboratoriais, radiológicos e cintilográficos para investigação etiológica da Síndrome do LC e DRGE. A técnica fisioterápica denominada aceleração de fluxo expiratório (AFE) foi realizada antes e após tratamento com cisaprida. O tempos totais de RGE (TTRGE), primeiramente durante a cintilografia basal, e em seguida, durante a AFE, foram analisados e somados, para cada topografia esofágica.

Resultados: a cisaprida diminuiu o TTRGE, com significância estatística somente no terço superior do esôfago $(\mathrm{p}<0,05)$ e não influenciou o TTRGE durante a AFE. Após tratamento com cisaprida, a AFE causou aumento do TTRGE nas topografias esofágicas média e superior, porém sem significância estatística. Lactentes com DRGE, antes do tratamento com cisaprida, tiveram menor TTRGE em esôfago distal durante a AFE $(\mathrm{p}<0,05)$. Após tratamento com cisaprida, essa significância estatística deixou de existir. Os LC sem DRGE também apresentaram menor TTRGE em esôfago distal durante a AFE $(p<0,05)$. Os LC com DRGE apresentaram maior TTRGE em esôfago distal $(\mathrm{p}<0,05)$, antes e após tratamento com cisaprida, tanto durante a cintilografia basal como durante a AFE.

Conclusão: a cisaprida foi eficaz na diminuição do TTRGE em LC com DRGE, principalmente no terço superior do esôfago. A fisioterapia respiratória, segundo a técnica de AFE, foi potencialmente refluxogênica. Outros estudos são necessários para investigar os efeitos da fisioterapia respiratória segundo posições corporais.

J Pediatr (Rio J) 2001; 77 (5):393-400: fisioterapia respiratória, síndrome do lactente chiador, refluxo gastroesofágico, cintilografia.

\begin{abstract}
Objective: to evaluate the effect of cisapride and chest physical therapy on the gastroesophageal reflux of wheezing babies.

Methods: we prospectively assessed the presence of technetium $\left({ }^{99} \mathrm{Tc}\right)$ in the upper, middle, and lower esophagus of 25 wheezing babies (13 with GERD and 12 without GERD) using scintigraphy. Both groups underwent clinical investigation, including laboratory, $\mathrm{X}$-ray and scintigraphy tests, for the etiology of the wheezing baby syndrome (WBS) and GERD. Expiratory Flow Acceleration (EFA) was performed before and after treatment with cisapride. The total time of GER episodes was accounted for each portion of the esophagus during scintigraphy and during EFA.

Results: cisapride significantly reduced the total reflux time in the upper esophagus $(\mathrm{P}<0.05)$, but showed no influence during EFA. After cisapride therapy, EFA increased the total reflux time in the upper and medium esophagus; however, no statistical significance was found. Infants with GERD presented a shorter total reflux time in the distal esophagus $(\mathrm{P}<0.05)$ during EFA. After cisapride treatment, no statistical significance was found. Infants without GERD also presented reduced total reflux time in the distal esophagus during EFA $(\mathrm{P}<0.05)$. Those with GERD had increased total reflux time in the distal esophagus $(\mathrm{P}<0.05)$ before and after cisapride treatment during EFA and scintigraphy.

Conclusions: cisapride was effective in reducing the total reflux time, mainly in the upper esophagus. EFA apparently increased the number of episodes of GER, without achieving statistical significance. Further studies are necessary to investigate the effects of chest physical therapy according to body positions.
\end{abstract}

J Pediatr (Rio J) 2001; 77 (5):393-400: chest physical therapy, wheezing babies, gastroesophageal reflux, scintigraphy.

1. Fisioterapeuta responsável pelo Serviço de Fisioterapia do Depto. de Pediatria da Fac. de Ciências Médicas (FCM) da Univ. Estadual de Campinas (Unicamp). 2. Médico(a) Assistente do Departamento de Radiologia e Medicina Nuclear da FCM Unicamp.

3. Professor Titular do Departamento de Radiologia e Medicina Nuclear da FCM Unicamp.

4. Professor Assistente Doutor do Departamento de Pediatria da FCM Unicamp, Pesquisador do Centro de Investigação em Pediatria (CIPED) da FCM Unicamp. Trabalho apresentado como Dissertação de Mestrado de M.A.G.O. Ribeiro no Curso de Pós-Graduação em Farmacologia da FCM Unicamp. 


\section{Introdução}

Dados recentes ${ }^{1}$ mostram que até $25 \%$ dos lactentes apresentam sibilância recorrente, constituindo um grande contigente de atendimento pediátrico ${ }^{2}$. Existem vários mecanismos e características que constituem fatores de risco para a sibilância nesta faixa etária como a flacidez e o pequeno calibre das vias aéreas, broncoespasmo, acúmulo de secreções e inflamação. As enfermidades mais freqüentemente envolvidas são atopia, infecção viral, doença do refluxo gastroesofágico (DRGE) e, em outros casos, a presença de corpo estranho ou compressão extrínseca das vias aéreas ${ }^{1,2}$. A expressão clínica da Síndrome do Lactente Chiador (SLC) é a sibilância contínua ou recidivante, que reflete a obstrução das vias aéreas.

O refluxo gastroesofágico (RGE) é o trânsito retrógrado e involuntário do conteúdo gástrico para o esôfago, podendo manifestar-se ou não com regurgitação ou vômito de saliva, alimentos, secreção gástrica, secreção biliar e/ou pancreática $^{3-7}$. O RGE pode ser fisiológico em qualquer indivíduo. O termo doença do refluxo gastroesofágico (DRGE) é utilizado para descrever o amplo espectro de distúrbios causados pelo $\mathrm{RGE}^{3}$. A distinção entre RGE fisiológico e DRGE é feita em função da quantidade de RGE observado, sendo que a DRGE se caracteriza por aumento na freqüência, intensidade e duração dos episódios de RGE, com danos à mucosa do esôfago e/ou do trato respiratório ${ }^{6}$. É a desordem mais frequiente do esfincter esofágico inferior (EEI) e deve ser considerada como causa de doença respiratória não controlada, incluindo a sibilân$\mathrm{cia}^{6,8}$.

A relação entre a SLC e RGE é complexa e não consensual $^{3-5}$. A maior prevalência de DRGE em LC não indica por si só uma relação causal. A DRGE pode causar ou agravar a obstrução brônquica, por meio de aspiração, reflexo vagal, aumento da reatividade brônquica e liberação de taquicinicas. A mecânica pulmonar alterada no LC (tosse, aumento do gradiente de pressão transdiafragmática, retificação do diafragma pela hiperinsuflação) e o uso de drogas refluxogênicas (metilxantinas e simpatomiméticos sistêmicos) são fatores de risco para a DRGE ${ }^{5,9}$.

O diagnóstico da DRGE deve ser confirmado por pelo menos dois métodos diagnósticos dentre estudo radiológico, cintilográfico ou ultrassonográfico ${ }^{10-12}$. Adota-se este critério quando não é possível realizar a monitorização prolongada do $\mathrm{pH}$ esofágico, considerado o melhor método diagnóstico para a DRGE ${ }^{13}$.

O tratamento da DRGE envolve medidas clínicas (decúbito elevado, dieta e farmacoterapia) e cirúrgicas ${ }^{6,14}$, tendo como objetivo, diminuir os fatores agressivos e aumentar os fatores protetores da mucosa esofágica ${ }^{13-17}$. Dentre as drogas utilizadas para o tratamento da DRGE, a cisaprida é o monoidrato de cis-4-amino-5-cloro-N-1-3-4- (fluorofenoxi-propil) 3-metoxi 4-piperidinil 3-metoxibenzamida. Seu principal mecanismo de ação é aumentar a liberação de acetilcolina nas terminações nervosas pós-ganglionares do trato gastrintestinal ${ }^{18,19}$. Seu efeito anti-RGE decorre do aumento da contração muscular do esôfago, do estômago e do tônus do EEI, sendo capaz de coordenar a motilidade antropiloro duodenal, acelerando o esvaziamento gástrico e impedindo a estase de conteúdo gástrico ${ }^{15,18}$. Não afeta a secreção gástrica, não estimula receptores dopaminérgicos e não apresenta atividades sobre o sistema nervoso central $^{20,21}$. Apesar das dúvidas recentes sobre sua segurança, a European Society of Pediatric Gastroenterology, Hepatology and Nutrition ${ }^{9}$ recomenda seu uso, com exceção dos pacientes que apresentam arritmias cardíacas, distúrbios hidroeletrolíticos, insuficiência hepática ou que recebam drogas que interfiram com citocromo $\mathrm{P} 450$, como os antifúngicos azoles (cetaconazol, miconazol e itraconazol) e os antibióticos macrolídeos (eritromicina, claritromicina e troleandromicina) $)^{4,7,8,13,19,22}$.

Muitas crianças que desenvolvem sibilância e obstrução de vias aéreas por hipersecreção brônquica são freqüentemente encaminhadas ao setor de fisioterapia respiratória do Hospital de Clínicas da Unicamp. Esta terapia tem por objetivo aumentar a depuração mucociliar, desobstruir as vias aéreas e facilitar a ventilação e as trocas gasosas ${ }^{23}$. É uma terapia complementar para atelectasia, bronquiectasia e desordens respiratórias complicadas por excesso e/ou retenção de secreção brônquica e pulmonar como a fibrose cística, SLC e bronquite crônica ${ }^{24-26}$.

Entretanto, existem poucos estudos na literatura abordando os riscos e benefícios da fisioterapia respiratória na SLC, bem como faltam normatizações de condutas fisioterapêuticas no tratamento de LC com ou sem DRGE. O objetivo deste estudo foi avaliar o efeito da cisaprida e da fisioterapia respiratória sobre o RGE em pacientes com SLC.

\section{Métodos}

Foram selecionados LC atópicos matriculados no Ambulatório de Alergia-Imunologia e Pneumologia Pediátrica do Hospital das Clínicas da Unicamp. Os critérios de inclusão foram idade superior a 3 mêses de vida, ocorrência de pelo menos 3 episódios de sibilância ou 1 episódio contínuo de sibilância com duração superior a trinta dias ${ }^{2}$, cujo diagnóstico de atopia levou em conta a história clínica, níveis de IgE sérica acima do percentil 95 para a idade, hemograma com $5 \%$ ou mais de eosinófilos e história familiar de atopia (pai ou mãe) ${ }^{27}$. Os pais concordaram em participar da pesquisa e assinaram o termo de consentimento esclarecido aprovado pelo Comitê de Ética em Pesquisa da Faculdade de Ciências Médicas da Unicamp.

Os pacientes foram avaliados prospectivamente e divididos em dois grupos. O primeiro grupo foi constituído por $\mathrm{LC}$ atópicos que tiveram DRGE confirmada ao raio $\mathrm{X}$ de esôfago, estômago e duodeno contrastado com bário (RxEED) e cintilografia para pesquisa de RGE, apresentando 
episódios de RGE nas topografias distal, média e superior do esôfago. Tais pacientes receberam cisaprida durante 4 a 8 meses (média de 6 meses), administrada 3 vezes ao dia, na dose de $0,2 \mathrm{mg} / \mathrm{kg} / \mathrm{dose}$. O segundo grupo foi constituído de LC atópicos que apresentaram RxEED e cintilografia negativos ou com raros episódios de RGE somente no esôfago distal, não sendo, portanto, considerados portadores de DRGE. Os dois grupos foram submetidos a investigação clínica, laboratorial, radiológica e cintilográfica para investigação etiológica da SLC, conforme rotina do Serviço de Alergia-Imunologia e Pneumologia Pediátrica da FCMUnicamp, incluindo hemograma, dosagem de imunoglobulinas séricas (IgA, IgG, IgM, IgE), teste de Mantoux e dosagem de sódio e cloro no suor.

Foram excluídos os LC que tiveram contra-indicado o uso da cisaprida ${ }^{7}$ ou que fossem portadores de desnutrição, baixa estatura ou baixo peso, infecções congênitas, tuberculose, fibrose cística, cardiopatias, doenças genéticas, retardo de desenvolvimento neuropsicomotor, imunodeficiência primária ou secundária ou outras doenças crônicas conhecidas. Também foram excluídos aqueles que apresentaram situações que contra-indicassem a fisioterapia respiratória, como pneumotórax, pneumatocele, derrame pleural, insuficiência respiratória aguda e abscesso pulmonar. Foi permitido o uso de beta agonistas por via inalatória nas crises de obstrução brônquica. Nenhum paciente estava em uso corrente de beta agonistas inalatórios na semana do estudo cintilográfico. Os pacientes que estavam em crise e necessitaram usar corticosteróides ou broncodilatadores por via sistêmica foram excluídos do estudo.

\section{Estudo Radiológico}

O RxEED foi executado segundo os critérios de McCauley e col. ${ }^{28}$, adaptados pelo Departamento de Radiologia da FCM-Unicamp. Após jejum de 4 horas, os LC receberam solução contendo bário, em quantidade suficiente para encher o estômago. Em seguida, foram observados à fluoroscopia intermitente durante 5 minutos, quando o examinador obteve radiografias que documentaram as imagens radiológicas mais relevantes. Foram observados em decúbito dorsal, dando-se ênfase para a anatomia e funcionamento do esôfago; e na posição oblíqua posterior direita, dando-se ênfase para a anatomia e funcionamento do estômago e duodeno. Foram valorizados, na observação radiológica, o clearence esofágico e a topografia esofágica atingida pelos episódios de RGE, permitindo classificá-los em grau I (esôfago distal), grau II (esôfago médio) e grau III (esôfago superior).

\section{Estudo Cintilográfico}

Os exames cintilográficos foram realizados no Serviço de Medicina Nuclear do Hospital de Clínicas da FCM Unicamp em duas etapas: primeiramente o estudo basal e a seguir, durante a fisioterapia respiratória. Após jejum de 6 horas, cada lactente recebeu a refeição de prova contendo $18,5 \mathrm{MBq}(0,5 \mathrm{mCi})$ de enxofre coloidal- ${ }^{-99} \mathrm{mTc}$, adicionados ao volume de leite habitualmente ingerido, em sua própria mamadeira. Os pacientes foram posicionados em decúbito dorsal horizontal, com o colimador na projeção anterior de tórax e terço superior de abdome. Utilizou-se colimador de baixa energia e alta sensibilidade. Durante a cintilografia basal, foram adquiridas uma imagem a cada 2 segundos por 15 minutos. Em seguida, durante a fisioterapia respiratória, repetiu-se a aquisição de uma imagem a cada 2 segundos por 15 minutos.

Os episódios de RGE foram analisados segundo a topografia esofágica atingida (terços distal, médio ou superior) e sua duração em segundos, primeiramente durante a cintilografia basal e, em seguida, durante a fisioterapia respiratória. As imagens obtidas durante esses dois períodos foram armazenadas em computador e analisadas individualmente. O tempo total dos episódios de RGE (TTRGE) foi somado para cada topografia esofágica (distal, média e superior), e os resultados expressos como TTRGE, em segundos, de RGE observado, para cada lactente estudado. A pesquisa de aspiração pulmonar foi realizada 4 e 24 horas após o início do estudo.

\section{Fisioterapia Respiratória}

Utilizou-se a técnica de aceleração de fluxo expiratório (AFE), com manobras passivas, ou seja, sem a colaboração voluntária do lactente, com o objetivo de aumentar o tempo e a velocidade do fluxo expiratório. Esta técnica emprega duas modalidades de velocidade de fluxo: AFE lenta e AFE rápida ${ }^{29}$. A técnica foi executada durante 15 minutos consecutivos, após a cintilografia basal. Realizou-se movimento torácico expiratório sincronizado, com as mãos, no início do platô inspiratório, sem ultrapassar os limites fisiológicos expiratórios da criança. $\mathrm{O}$ fluxo aéreo induzido tem velocidade superior à da expiração normal e próxima à da tosse. Desse modo, a AFE apenas potencializa a fisiologia pulmonar normal, através de variações de fluxos aéreos, para a desobstrução brônquica e homogeneização da ventilação pulmonar $^{30}$. As mãos da fisioterapeuta se posicionaram uma no tórax (mão da pressão expiratória) e a outra nas costelas inferiores, minimizando o aumento da pressão abdominal e, conseqüentemente, episódios de RGE iatrogênicos. Apesar de esta técnica permitir vários posicionamentos, utilizou-se somente o decúbito supino, sendo este o decúbito rotineiramente utilizado durante a cintilografia basal e procedimentos fisioterápicos ${ }^{29,30}$.

\section{Análise estatística}

Os TTRGE observados nos terços esofágicos distal, médio e superior, foram contabilizados para cada lactente. A constituição dos grupos para análise estatística levou em conta a classificação dos pacientes segundo os grupos de LC com ou sem DRGE, a topografia esofágica atingida pelo 
RGE, presença ou ausência da terapia com cisaprida e da fisioterapia respiratória. Após estudo estatístico descritivo, os TTRGE foram comparados por meio dos testes não paramétricos de Wilcoxon ou Mann-Whitney, considerando-se o valor de $\mathrm{p}<0,05$ como estatisticamente significativo ${ }^{31}$.

\section{Resultados}

\section{Casuística}

O primeiro grupo de pacientes foi constituído por 13 LC atópicos (10 meninos e 3 meninas) cuja média de idade foi 9,8 meses e a média de peso foi $11,6 \mathrm{~kg}$; todos tiveram DRGE confirmada ao raio $\mathrm{X}$ de esôfago, estômago e duodeno contrastado com bário (RxEED) e cintilografia para pesquisa de RGE, apresentando episódios de RGE nas topografias distal, média e superior do esôfago. O segundo grupo de pacientes foi constituído de 12 LC atópicos (6 meninos e 6 meninas) cuja média de idade foi 8,7 meses e a média de peso foi $10,7 \mathrm{~kg}$; todos apresentaram RxEED e cintilografia negativos ou com raros episódios de RGE somente no esôfago distal, não sendo, portanto, considerados portadores de DRGE. Ambos os grupos foram semelhantes quanto à gravidade da SLC, tendo usado, esporadicamente, apenas broncodilatadores inalatórios de curta duração.

\section{Protocolo experimental}

Durante a cintilografia basal, observou-se que, após o tratamento com cisaprida, houve diminuição do TTRGE nos LC com DRGE, nas três topografias esofágicas, ocorrendo significância estatística somente nos resultados referentes ao terço esofágico superior (Figura $1, \mathrm{p}<0,05, \mathrm{n}=13$, teste de Wilcoxon). Durante a fisioterapia respiratória, observou-se que o tratamento com cisaprida não influenciou no TTRGE desses pacientes, em quaisquer das topografias esofágicas (Figura 1, p>0,05 em todas situações, $\mathrm{n}=13$, teste de Wilcoxon).

Antes de receber tratamento com cisaprida, observouse que os LC com DRGE apresentaram menor TTRGE nas topografias esofágicas distal e média, durante a fisioterapia respiratória, realizada após cintilografia basal, ocorrendo significância estatística somente no TTRGE do terço esofágico distal (Figura 1, $\mathrm{p}<0,05, \mathrm{n}=13$, teste de Wilcoxon). Em contrapartida, após serem tratados com cisaprida, observou-se que os LC com DRGE apresentam tendência de aumento do TTRGE nas topografias esofágicas média e superior, entretanto, sem significância estatística (Figura 1, p>0,05 em todas situações, $n=13$, teste de Wilcoxon), resultados que nos pareceram um tanto contraditórios, visto que a literatura considera a fisioterapia respiratória um fator refluxogênico, e o tratamento com cisaprida, um recurso para seu controle.

Com o objetivo de esclarecer esta aparente discrepância, confrontamos estes resultados do grupo dos LC com

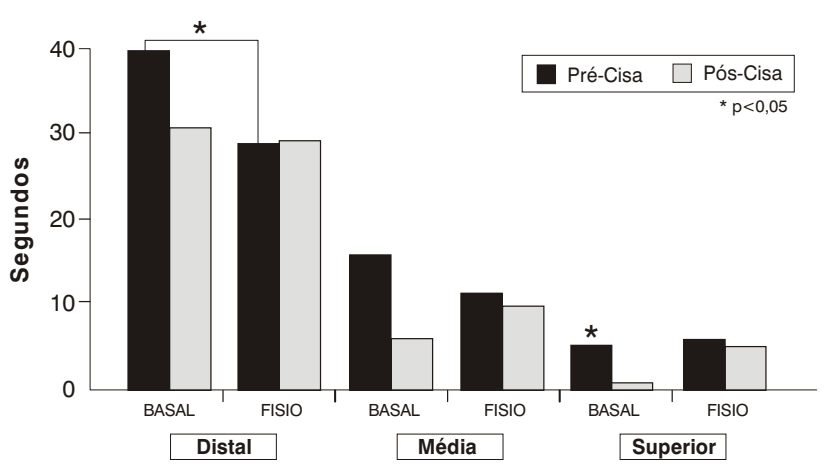

Figura 1 - Média do tempo total de refluxo gastroesofágico (TTRGE) observado no esôfago superior, médio e distal segundo avaliação cintilográfica de lactentes chiadores (LC) com doença do refluxo gastroesofágico (DRGE) antes e após tratamento com cisaprida, primeiramente durante cintilografia basal e em seguida, durante fisioterapia respiratória sob a técnica de aceleração de fluxo expiratório (AFE)

DRGE, antes e após terapia com cisaprida, registrados durante cintilografia basal ou durante a fisioterapia respiratória, com os resultados do grupo de LC sem DRGE e, portanto, sem tratamento com cisaprida, registrados durante cintilografia basal ou fisioterapia respiratória. Os LC sem DRGE também apresentaram menor TTRGE em esôfago distal, durante a fisioterapia respiratória, feita após cintilografia basal de 15 minutos (Figura 2, $\mathrm{p}<0,05, \mathrm{n}=12$, teste de Wilcoxon). Antes de serem tratados com cisaprida, observou-se que o TTRGE em esôfago distal dos LC com DRGE $(n=13)$ foi significativamente maior que o dos LC sem DRGE $(n=12)$, durante cintilografia basal ou fisioterapia respiratória (Figura 2, p <0,05, teste de Mann-Whitney). Mesmo após receber tratamento com cisaprida, observouse que o TTRGE em esôfago distal dos LC com DRGE $(n=13)$ permaneceu significativamente maior que o dos LC sem DRGE ( $n=12)$, durante cintilografia basal ou fisioterapia respiratória (Figura 2, p<0,05, teste de Mann-Whitney).

\section{Discussão}

O RGE é a desordem mais importante do esôfago e do EEI na infância. É considerado fator de risco para a ocorrência de infecções respiratórias de repetição, crises de asma e piora de pacientes com pneumopatia crônica. Constitui o diagnóstico diferencial ou associado mais procurado nos LC, cuja investigação é rotina obrigatória em nosso meio $^{3}$.

A definição de SLC utilizada no presente estudo ${ }^{2}$ é semelhante àquelas utilizadas por outros autores ${ }^{18,32-36} \mathrm{e}$ foi escolhida por ser a mais citada e aceita na literatura brasileira. 
Dentre os $25 \mathrm{LC}$ atópicos integrantes deste estudo (13 com DRGE e 12 sem DRGE), nossa maior prevalência de pacientes do sexo masculino (64\%) está em concordância com os dados da literatura ${ }^{1}$. Poucos estudos comparam as influências raciais, idade de início e gravidade da sintomatologia em LC. Apesar de existirem fenótipos de LC classificados antes e após 3 anos de idade ${ }^{1}$, abaixo desta faixa etária, este evento ainda não foi estudado, a não ser quanto ao diagnóstico diferencial. Na população deste estudo, as características clínicas foram homogêneas, e a casuística envolveu LC atópicos, mas com manifestações diferentes quanto ao RGE documentado com dois tipos exames.

A análise dos resultados (Figuras 1 e 2) sugere que o TTRGE, nas diversas topografias esofágicas, é inversamente proporcional à distância do EEI, em todas as situações estudadas (pré e pós-tratamento com cisaprida; durante cintilografia basal ou fisioterapia respiratória). Na patogenia do RGE esta observação ainda não é completamente compreendida, e mesmo estudos por meio de pHmetria não conseguiram explicar o que é mais patogênico: muitos episódios de RGE no esôfago distal ou poucos, mas no esôfago proximal ${ }^{15}$.

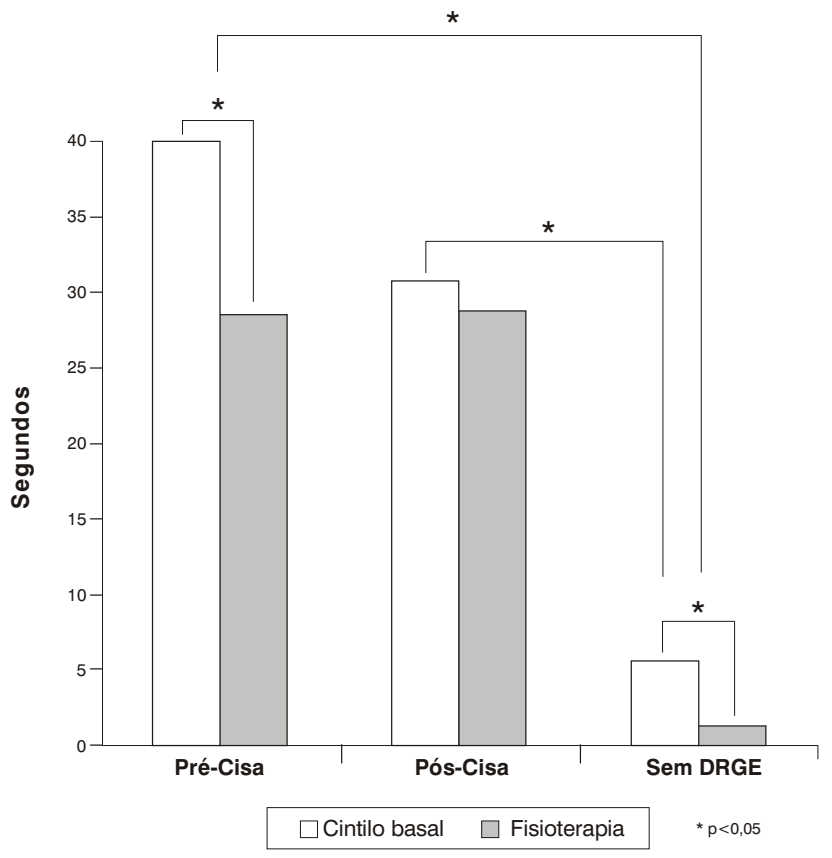

Figura 2 - Média do tempo total de refluxo gastroesofágico (TTRGE) observado no esôfago distal, segundo avaliação cintilográfica de lactentes chiadores (LC) com ou sem doença do refluxo gastroesofágico (DRGE) antes e após tratamento com cisaprida, primeiramente durante cintilografia basal e em seguida, durante fisioterapia respiratória sob a técnica de aceleração de fluxo expiratório (AFE)
Agentes pró-cinéticos como a cisaprida aceleram o esvaziamento gástrico e, com isso, minimizam as chances de ocorrência de episódios de RGE ${ }^{15}$. Nossos resultados mostram que a cisaprida diminuiu o TTRGE em todas as topografias esofágicas no grupo de LC com DRGE, embora com significância estatística somente nos resultados referentes ao terço superior do esôfago (Figura 1). Esses achados mostram uma nova particularidade desse medicamento, que é a redução dos episódios de RGE no esôfago superior, responsáveis pelos mecanismos de sibilância secundária à aspiração ${ }^{4}$.

Em contrapartida, durante a fisioterapia respiratória, realizada após os 15 minutos da cintilografia basal, o tratamento com a cisaprida não influenciou no TTRGE dos LC com DRGE nas três topografias esofágicas (Figura 1), sugerindo que a estimulação física possa ter efeito refluxogênico, não antagonizado pela cisaprida. Ressaltamos que os mecanismos que regulam o tônus do EEI dependem de forças de abertura e forças de fechamento ${ }^{37}$. Embora os mecanismos pelos quais a fisioterapia respiratória interfere no funcionamento do EEI não sejam no momento conhecidos, consideramos intrigante que a fisioterapia possa antagonizar a mais potente droga pró-cinética utilizada em pediatria.

Embora seja um agente pró-cinético efetivo para o tratamento de $\mathrm{RGE}^{7}$, nossos dados mostram que, mesmo após receberem o tratamento com cisaprida, os LC com DRGE apresentam um TTRGE significativamente maior no esôfago distal, em comparação com os LC sem DRGE (Figura 2). Essa observação indica que a cisaprida, nos LC com DRGE, não estabeleceu o mesmo padrão de RGE no esôfago distal, observado nos LC sem DRGE.

Durante a fisioterapia repiratória nos LC com DRGE, antes do tratamento com cisaprida (Figura 1), observamos que o TTRGE no esôfago distal foi significativamente menor, quando parte do esvaziamento gástrico já tinha ocorrido $(\mathrm{p}<0,05)$, redução de tempo que não ocorreu nas topografias esofágicas média e superior. É provável que a fisioterapia respiratória tenha pouca importância sobre os episódios de RGE no esofágico distal como pode ser evidenciado nesta mesma figura, onde o estímulo fisioterápico aumentou o TTRGE nos terços esofágicos médio e superior, mesmo após o tratamento com cisaprida.

A fisioterapia respiratória clássica consiste na utilização de estímulos físicos na região torácica e de posturas corporais somadas a forças gravitacionais, para favorecer e otimizar a drenagem de secreções brônquicas impactadas ${ }^{25,26,38}$. Vandenplas e col. ${ }^{39}$, utilizando as técnicas convencionais de fisioterapia respiratória como drenagem postural na posição corporal de Trendelenburg, percussão, vibração e tosse provocada, observaram episódios de RGE em três grupos de lactentes: com RGE, com insuficiência respiratória aguda e grupo controle. Não houve correlação temporal entre a tosse provocada e os episódios de RGE. Esse estudo não fez comparações com outras posições corporais. 
Sabe-se que a postura corporal tem impacto substancial na frequiência do relaxamento transitório do EEI. Esses relaxamentos são tidos como mecanismos determinantes no aumento do RGE em pacientes que desenvolvem esofagite, mais do que a baixa pressão do EEI ou o aumento da pressão intragástrica $^{40}$. A posição corporal é uma preocupação constante para a desobstrução brônquica em lactentes com $\mathrm{RGE}^{38}$. Alguns autores contra-indicam a drenagem postural em pacientes com RGE e orientam a elevação da cabeça a 30 graus como parte essencial do tratamento não medicamentoso ${ }^{6,13}$. Os estudos são controversos quanto à utilização do decúbito de Trendelenburg como recurso para a desobstrução brônquica em pacientes com $\mathrm{RGE}^{41,42}$. Ao contrário dos estudos de Vandenplas ${ }^{39}$, não realizamos manobras de compressão abdominal para favorecimento da tosse e não encontramos aumento de RGE nos LC sem DRGE.

A AFE tem resultados clínicos positivos quando aplicada em LC com hipersecreção brônquica. Esta técnica, realizada com as mãos, pode controlar os estímulos, a velocidade do fluxo e o tempo do período expiratório, podendo ser ajustada conforme a necessidade de mobilização de secreções, se em vias aéreas centrais ou periféricas. Os estímulos empregados devem ser adequados ao tamanho do tórax do paciente pediátrico ${ }^{29,30}$. Em nosso trabalho, para manter a qualidade, a AFE foi realizada sempre pelo mesmo profissional.

Como qualquer outra manobra desobstrutiva em lactentes, o emprego da AFE pode aumentar a pressão intratorácica negativa, pois, com frequiência, aumenta a inspiração, a tosse e provoca choro. Ressaltamos que este estímulo não provocou aspiração pulmonar em nenhum dos lactentes estudados, mesmo estando em decúbito supino e no período pós-prandial, reforçando a segurança desta técnica. Outros estudos deverão ser realizados para verificar a eficácia da AFE comparada às outras técnicas desobstrutivas convencionais.

Uma certa quantidade de episódios de RGE é um fenômeno normal em lactentes abaixo de 1 ano de idade, os quais podem refluir durante mais de 2 horas após as refeições ${ }^{43}$. Sendo a cintilografia um teste pós-prandial, esta pode detectar tanto RGE fisiológico quanto patológico, levando a dificuldades na decisão do tratamento, sem o apoio de outro método diagnóstico. Em nosso estudo, o diagnóstico de RGE foi documentado segundo alterações encontradas em dois métodos diagnósticos: RxEED e cintilografia. O RGE pós-prandial, embora ocorra em pessoas saudáveis, pode ser responsável por doença pulmonar gra$\mathrm{ve}^{44} \mathrm{e}$ eventos ameaçadores da vida ${ }^{10,13,45}$. Alguns autores ressaltam que os episódios de aspiração maciça podem ocorrer no período pós-prandial, invalidando a idéia de que o RGE pós-prandial é sempre fisiológico ${ }^{32,46}$.

A cintilografia pode ser realizada por períodos prolongados, sem aumentar a exposição do paciente à radiação; não é invasiva ${ }^{47,48}$; não requer sedação ${ }^{49}$; é bem tolerada; e rapidamente realizada ${ }^{35}$. Permite documentar aspiração pulmonar $^{50}$, determina características do RGE tais como volume, clearance, tempo ${ }^{50-52}$ e, conseqüentemente, avalia o efeito de drogas pro-cinéticas para o tratamente de $\mathrm{RGE}^{51}$. Latini e col. ${ }^{52}$ ressaltam que a cintilografia pode ser considerada efetiva e confiável para diagnosticar RGE.

Alguns autores ${ }^{44,51-53}$ consideram que a cintilografia é superior à pHmetria para ao diagnóstico de RGE, enquanto outros autores consideram o inverso ${ }^{3,54}$. Vandenplas e col. propõem que estes dois procedimentos exploram diferentes condições, sendo ambos úteis na investigação de RGE em lactentes $^{55}$. Tais controvérsias se explicam pela grande variação na padronização dessas técnicas. Shay e col. ${ }^{41} \mathrm{e}$ Vandenplas e col. ${ }^{55}$ compararam essas técnicas durante o período pós-prandial, mostrando maior sensibilidade da cintilografia para detectar RGE pós-prandial. A explicação desse fato é que o estômago é distendido e tem seu $\mathrm{pH}$ neutralizado pela refeição ingerida, sendo o RGE detectado pela cintilografia, mas não pela $\mathrm{pHmetria,} \mathrm{incapaz} \mathrm{de} \mathrm{detec-}$ tar RGE neutros, ou seja, a cintilografia não depende do $\mathrm{pH}$ do conteúdo gástrico para detectar RGE.

A SLC e a DRGE continuam sendo duas entidades de muito interesse e com muitas dúvidas a serem esclarecidas. A relação de causa e efeito entre as duas ainda é controversa, devendo-se comprovar, no futuro, os mecanismos envolvidos. Concluímos que a cisaprida foi eficaz em diminuir o TTRGE, principalmente no esôfago superior de LC com DRGE, e que a AFE aparentemente aumenta o número de episódios de RGE, mas sem causar aspiração pulmonar ou outras repercussões clínicas.

\section{Referências bibliográficas}

1. Martinez FD, Wright AL, Taussig LM, Holdeberg CJ, Halonen M, Morgan WJ, et al. Asthma and wheezing in the six years of live. N Engl J Med 1995; 332: 133-8.

2. Rozov T, Bresolin AMB. A Síndrome do Lactente Chiador. In: Sampaio MMSC, Grumach AS. Alergia e Imunologia em Pediatria. São Paulo: Ed. Sarvier; 1992. p.51-9.

3. Quintela, TMM. Avaliação de refluxo gastroesofágico na morbidade de lactentes chiadores atópicos e não atópicos [tese de doutorado]. Campinas: Unicamp; 1997.

4. Brown P. Medical management of gastroesophageal reflux. Curr Opin Pediatr 2000; 12:247-50.

5. Eid NS, Shephard RW, Thomson MA. Persistent wheezing and gastroesophageal reflux in infants. Pediatr Pulmonol 1994; 18:39-44.

6. Vandenplas Y, Ashkenazi A, Belli D, Boige N, Bouquet J, Cadranel S, et al. A proposition for the diagnosis and treatment of gastroesophageal reflux disease in children: a report from a working group on gastroesophageal reflux disease. Eur J Pediatr 1993; 152: 704-11.

7. Vandenplas Y, Belli DC, Benatar A, Cadranel S, Cucchiara S, Dupont $\mathrm{C}$, et al. The role of cisapride in the treatment of pediatric gastroesophageal reflux. The European Society of Pediatric Gastroenterology, Hepatology and Nutrition. J Pediatr Gastroenterol Nutr 1999; 28:518-28. 
8. Vandenplas Y. Asthma and gastroesophageal reflux. J Pediatr Gastroenterol Nutr 1997; 24: 89-99.

9. De Campos-Mello AL. Avaliação do refluxo gastroesofágico em crianças e adolescentes asmáticos atópicos graves com e sem pneumonias recidivantes [dissertação de mestrado]. Campinas: Unicamp; 1999.

10. Euler AR, Byrne WJ, Ament ME, Fonkalsrud EW, Strobel CT, Ssiegel SC. Recurrent pulmonary disease in children: a complication of gastroesophageal reflux. Pediatrics 1979; 63: 47-51.

11. Berquist WE, Rachelefsky GS, Kadden M, Siegel SC, Katz RM, Fonkalsrud EW. Gastroesophageal reflux-associated recurrent pneumonia and chronic asthma in children. Pediatrics 1981; 68: 29-35.

12. Meyers W, Roberts CC, Johnson DG, Herbst JJ. Value of tests for evaluation of gastroesophageal reflux in children. J Pediatr Surg 1985; 20: 515-20.

13. Vandenplas Y, Hegar B. Diagnosis and treatment of gastrooesophageal reflux disease in infants and children. J Gastroenterol Hepatol 2000; 15:593-03.

14. Orenstein SR, Whitington FP, Orenstein DM. Positioning for prevention of infant gastroesophageal reflux. J Pediatrics 1983; 103: 534-37.

15. Hillemeier AC. Gastroesophageal reflux - Diagnostic and therapeutic approaches. Pediatr Clin North Am 1996;43:197-212.

16. Hare C, Halligan S, Bartram CI, Platt K, Raleigh G. Cisapride or metoclopramide to accelerate small bowel transit during barium follow-through examination? Abom Imaging 2000; 25:243-45.

17. Chen SD, Kao CH. Salivary function in patients with reflux esophagitis: effect of cisapride. J Nucl Med 1998; 39: 1440-52.

18. Vandenplas Y, Deneyer M. Gastroesophageal reflux incidence and respiratory dysfunction during sleep in infants: treatment with cisapride. J Pediatr Gastroenterol Nutr 1989; 8: 31-6.

19. Bohets H, Lavrijsen K, Hendrickx J, van Houdt J, van Genechten $\mathrm{V}$, Verbotven P, et al. Identification of the cytochrome P450 enzymes involved in the metabolism of cisapride: in vitro studies of potential co-medication interactions. Br J Pharmacol 2000; 129:1655-67.

20. Premiji SS, Paes B. Cisapride: the problem of heart. Neonatal Netw 1999; 18:21-5.

21. Schapira M, Henrion J, Heller FR. The current status of gastric prokinetic drugs. Acta Gastroenterologica Belgica 1990; 3: 446-57.

22. Michalets EL, Williams CR. Drug interactions with cisapride: clinical implications. Clin Pharmacokinet 2000; 39:49-75.

23. Hardy KA. A review of airway clearance: new techniques, indications, and recommendations. Respiratory Care 1994; 39: 440-55.

24. Prasad AS. Current concepts in physiotherapy. J R Soc Med 1993; 86:23-9.

25. Phillips GE, Pike SE. Holding the baby: head downward positioning for physiotherapy does not cause gastro-oesophageal. Eur Respir J 1998; 12: 954-57.

26. Flownfelter DL. Chest physiotherapy and pulmonary rehabilitation: an interdisciplinary approach. $2^{\mathrm{a}}$ ed. Year Book Medical Publishers 1987 INC.

27. Martinez FD. Recognizing early asthma. Allergy 1999; 54: 24-8.

28. McCauley RGR, Darling DB. Gastroesophageal reflux in children: a useful classification and reliable physiologic technique for its demonstration. Am J Roentgenol 1978; 130: 47-50.

29. Demont B, Escourrou CI, Vinçon C. Effets de la kinésithérapie respiratoire et des aspirations naso-pharyngées sur le reflux gastroesophagien chez l'enfant de 0 à 1 , avec et sans reflux pathologique. Archives Françaises de Pediatrie 1991; 48:621-25.
30. Barthe J, Binoche C, Brossard V. Pneumokinésithérapie. Paris: Doin; 1990

31. Bhattacharyya GH, Johson RA. In: Statistical concepts and methods. John Wiley \& Sons, Inc, New

32. Arasu TS, Wyllie R, Fitzgerald FJ. Gastroesophageal reflux in infants and children- comparative accuracy of diagnostic methods. The Journal of Pediatrics 1980:798-803.

33. Orenstein SR, Whitington FP, Orenstein D. The infant seat as treatment for gastroesophageal reflux. New Engl J Med 1983; 309: 760-3.

34. Cucchiara S, Staiano A. Cisapride for gastroesophageal reflux and peptic oesophagitis. Arch Dis Child 1987; 62: 454-7.

35. Shay SS, Abreu SH, Tusuchida MD. Scintigraphy in gastroesophageal reflux disease: a comparison to endoscopy, LESp, and 24-h score, as well as to simultaneous $\mathrm{pH}$ monitoring. Am J Gastroenterol 1992; 87: 1094-109.

36. Orenstein RS, Klein AK. Scintigraphy versus $\mathrm{pH}$ probe for quantification of pediatric gastroesophageal reflux: a study using concurrent multiplexed data and acid feeding. J Nucl Med 1993; 34: 1228-34.

37. Boix-Ochoa J. Address of honored guest: the physiologic approach to the management of gastric esophageal reflux. J Pediatric Surgery 1986; 12: 1032-9.

38. Button MB, Heine GR. Postural drainage and gastroesophageal reflux in infants with cystic fibrosis. Arch Dis Child 1998; 76: 148-50.

39. Vandenplas Y, Diericx A. Esophageal $\mathrm{pH}$ monitoring data during chest physiotherapy. J Pediatr Gastroenterol Nutr 1991; 13 : 23-6.

40. Mittal RK, Holloway RH, Penagini R,Blackchaw LA, Dent J. Transient lower esophageal sphincter relaxation. Gastroenterology 1995; 109: 601-10.

41. Foster AC, Voyles JB, Murphy AS. Twenty four hour pHmonitoring in children with cystic fibrosis: association of chest physical therapy to gastroesophageal reflux. Pediatr Res 1983: 17 Supp.ii, 188A.

42. Taylor AJ, Therelfall D. Postural drainage techniques and gastro-oesophageal reflux in cystic fibrosis. Lancet 1997; 349:1567-68

43. Vandenplas Y, Malfroot A, Dab I. Esophageal pH monitoring criteria in infants with recurrent respiratory disease. Pediatr Res 1998; 24: 417-21.

44. Oreinstein SR, Oreinstein DM. Gastroesophageal reflux and respiratory disease in children. J Pediatr 1988; 112: 847-58.

45. McCallum RW. Gastric emptying in gastroesophageal reflux and therapeutic role of prokinetic agents. Gastroenterol Clin North Am 1990; 19:551- 64.

46. Kaye AS, Siraj QH, Agnew J, Hilton A, Black C. Detection of early asymptomatic esophageal dysfunction in systemic sclerosis using a new scintigraphic grading method. J Rheumatol 1996; 23:297-01.

47. Fisher RS, Malmud LS, Roberts GS, Milmoe, G. Gastroesophageal scintiscanning to detect and quantitate gastroesophageal reflux. Gastroenterology 1976; 70:301-8.

48. Seibert CC, Byrne WJ, Euler AR, Latture T, Leach M. Gastroesophageal reflux: the acid test, scintigraphy or $\mathrm{pH}$ probe? Am J Roentgenol 1983; 140:1087-90.

49. Boonyaprapa S, Alderson PO, Garfinkel DJ, Chipps BE, Wagner HN JR. Detection of pulmonary aspiration in infants and children with respiratory disease: concise communication. J Nucl Med 1980; 21:314-18.

50. Tolia V, Kuhns LR, Kauffman RE. Comparison of simultaneous esophageal $\mathrm{pH}$ monitoring and scintigraphy in infants with gastroesophageal reflux. Am J Gastroenterol 1993; 88: 661-4. 
51. Washington N, Moss HA, Washington C, Greaves JL, Steele RJC, Wilson CG. Non-invasive detection of gastro-oesophageal reflux using an ambulatory system. Gut 1993; 34:1482-86.

52. Latini G, Del Vecchio A, De Mitri B, Giannuzzi R, Presta G, et al. Scintigraphic evaluation of gastroesophageal reflux in newborn. Pediatr Med Chir 1999; 21:115-17.

53. McVeach P, Houman-Giles R, Kemp A. Pulmonary aspiration studied by radionuclide milk scanning and barium swallow roentgenography. Am J Dis Child 1987; 141:917-21.

54. Fawcett HD, Hayden CK, Adams JC, Swischuk LE. How useful is gastroesophageal reflux scintigraphy in suspected childhood aspiration? Pediatr Radiol 1988; 18: 311-13.
55. Vandenplas Y, Derde MP, Piepsz A simultaneously performed esophageal $\mathrm{pH}$ monitoring and scintigraphy. J Pediatr Gastroenterol Nutr 1992; 14: 256-60.

Endereço para correspondência:

Dra. Maria Angela G. de O. Ribeiro

Rua Osório Alves, 612 - Bairro Cidade Universitária

CEP 13084-020 - Campinas, SP

Fone: (19) 3289.3874 - Fax: (19) 3788.8970

E-mail: ribeirojd@lexxa.com.br 